\title{
Pattern formation at cellular membranes by phosphorylation and dephosphorylation of proteins
}

Sergio Alonso

\begin{abstract}
We consider a classical model on activation of proteins, based in two reciprocal enzymatic biochemical reactions. The combination of phosphorylation and dephosphorylation reactions of proteins is a well established mechanism for protein activation in cell signalling. We introduce different affinity of the two versions of the proteins to the membrane and to the cytoplasm. The difference in the diffusion coefficient at the membrane and in the cytoplasm together with the high density of proteins at the membrane which reduces the accessible area produces domain formation of protein concentration at the membrane. We differentiate two mechanisms responsible of the pattern formation inside of living cells and discuss the consequences of these models for cell biology.
\end{abstract}

\section{Introduction}

Cascades of biochemical reactions and interactions regulate multiple processes inside living cells [1]. Proteins, enzymes and small molecules strongly interact and participate in genetic and metabolic networks.

Biochemical processes inside cells are highly non-linear and their dynamics complex. Two characteristics examples of such complexity in the cell are genetic regulatory networks and cell signalling [2]. Regulatory pathways involve different types of proteins, which control the transcription of the genes. They form the genetic networks in cell biology and governs processes at large times scales (hours). A protein that represses the transcription of its own gene is a simple example of a regulatory network. The repression produces a negative feedback and under certain conditions it induces a periodic synthesis of the protein [3].

Sergio Alonso

Departament de Física, Universitat Politècnica de Catalunya, Av. Dr. Marañon 44-50, 08028, Barcelona, Spain, e-mail: s.alonso@upc.edu 
On the other hand, interactions among different proteins can also produce complex biochemical oscillations of protein concentration inside signalling pathways [4]. Cell signalling controls the rapid response of the cell to external variations of the environment, and corresponds to fast processes at small time scales (minutes). One of the simplest components of such pathways are enzymatic reactions. An enzyme, is a protein which catalyzes and accelerates a particular biochemical reaction, which under other circumtances would be much slower [5]. An important characteristics of the enzyme is that it takes part in the reaction, however, after the reaction occurs, the enzyme is completely recovered and can catalyze again another reaction.

A phosphorylation reaction consists in the incorporation of a phosphate group to a certain protein, producing a phosphorylated version of the protein. Such type of biochemical reactions are catalyzed by kinases. Protein kinases are enzymes and the phosphorylation reaction is an enzymatic reaction. The opposite reaction is also possible. The dephosphorylation reaction removes the phosphate group from the phosphorylated protein, and it is catalyzed by the enzyme phosphatase. Protein kinases and phosphatases are particularly active in signalling processes. Important parts of biochemical pathways consist on multiple phosphorylations and dephosphorylations of diverse proteins [6].

When both processes occurs simultaneously, phosphorylation and dephosphorylation may control the activity of a particular protein in a pathway. Activation and deactivation are important mechanisms in the regulation of many cellular processes. Both reactions are usually described in well-mixed environments by the GoldbeterKoshland model of reciprocal covalent modifications [7]. The stiff response of the system to small changes in the kinase or the phosphatase concentrations makes the Goldbeter-Koshland mechanism a good model for activation of proteins in signalling pathways. The response of the model to the change of the enzyme concentration is stiff but monotonous and, therefore, no bistability or another kind of pattern formation mechanisms are accessible from this model. The complexity necessary for a non-monotonous behaviour can be incorporated by positive and negative interactions among the two versions of the proteins and the enzymes [8].

An alternative strategy for the formation of complex dynamics is the incorporation of spatial restrictions. Living cells are not always well-mixed environments and active or passive transport is crucial for the organization of some metabolic and genetic processes [9]. Furthermore, there is a high degree of compartmentalization in the cell and different types of proteins are located in different parts of the cell. Thus, the spatial aspects of the interior of the cells become relevant in intracellular communication [10] and self-organization may rule many processes in cell biology [11]. In particular, kinases and phosphatases may locate in opposite positions inside the cell, e.g. membrane/cytoplasm [12,13], nucleus/cytoplasm [14] or anterior/posterior $[15,16]$. It may produce the formation of spatial gradients in metabolic reactions. The formation of biochemical gradients may induce the polarization of the cell [17], and the definition of a preference direction for a posterior motion [18] or division [19] of a living cell.

The equations employed for the mathematical modeling of cell polarity typically consist in two components, a membrane protein with very slow diffusion in 
comparison with a second protein which diffuses faster through the cytoplasm. The interactions between these two components, which are highly non-linear, produce a Turing-like instability of the homogeneous solution [20,21, 22] or a wave-pinning dynamics due to the frustration under bistable conditions of the wave between both stable solution due to a mass-conservation condition [19, 23, 24, 25].

Here, we derive from the basis dynamics of enzymatic reactions a reactiondiffusion system of three equations representing the concentrations of the same protein at the membrane, phosphorylated and dephosphorylated in the cytoplasm. A similar set of equations have been previously successfully employed for the modeling of experimental observations on protein translocation results in an insulinsecreting cell $[26,27]$.

This chapter is organized as follows, first we review in Sec.2 the derivation of the simplest model on reciprocal covalent modification composed by the phosphorylation and dephosphorylation processes. Second, we introduce in Sec.3 the effects of compartemization and the effects of saturation at the membrane where the large amount of membrane proteins restricts the accessible area. Finally, in Sec. 4 the transport by diffusion is incorporated to the model of the biochemical reactions to generate the final reaction-diffusion model. The different mechanisms of pattern formation are described and analyzed.

\section{Modelling enzymatic kinetics}

The basis of any enzymatic reaction is the fast conversion of a substrate $S$ into a product $P$. One is tempt to consider the next linear conversion

$$
S+E \rightarrow P+E
$$

for the modeling of an enzymatic reaction, see the simple sketch in Fig.1(a) of the reaction in eq.(1). The velocity of reaction, which corresponds to the rate of production of $[P]$, has a simple linear relation with the concentration of the substrate and it is linearly proportional to the concentration of the enzyme:

$$
\frac{\partial[P]}{\partial t}=k[E][S]
$$

where $k$ is the rate of the reaction in eq.(1). This simple dynamics holds when the number of substrate molecules is small in comparison with the capacity of enzymes to induce the reactions. If the number of substrate molecules is large, there is a delay due to the lack of available enzymes to perform the reaction. In this case the linear approximation shown in eq.(1) is not correct. 
(a)

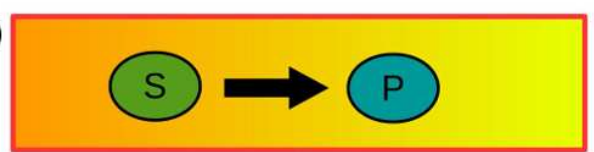

(b)

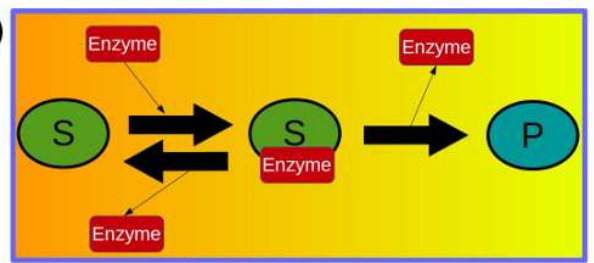

(c)

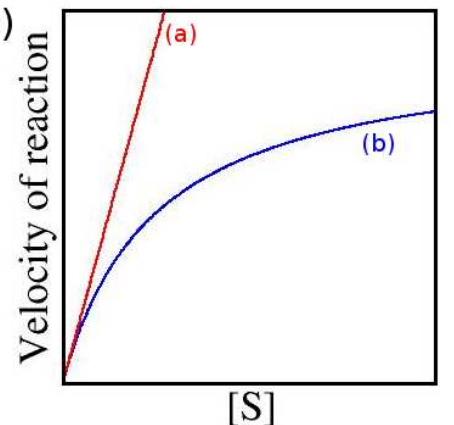

Fig. 1 (a) Sketch of the conversion of a subtract into a product through a linear reaction. (b) Sketch of the conversion of a substract into a product through an enzymatic reaction. (c) Dependence of the velocity of reaction in the concentration of the substrate for the two types of reactions.

\subsection{Michaelis-Menten model}

Although the number of enzymes is the same before and after a reaction event, it participates in the reaction. Enzymes change the structure of the substrate to enhance the affinity to generate the product. We consider an intermediate step: the enzyme reacts with the substrate giving rise to a complex molecule $C$. This complex state may react and give rise to the product together with the original enzyme, however, there is a small probability than the complex $C$ reacts in the opposite direction giving rise to the substrate and the enzyme. The three reactions together read:

$$
S+E \rightleftharpoons C \rightarrow P+E
$$

and a schematic description of the reaction is shown in Fig. 1(b). If we apply the law of mass action to the three reactions we obtain that the four concentrations follow [5]:

$$
\begin{aligned}
\frac{\partial[S]}{\partial t} & =k_{-1}[C]-k_{1}[S][E], \\
\frac{\partial[E]}{\partial t} & =\left(k_{-1}+k_{2}\right)[C]-k_{1}[S][E], \\
\frac{\partial[C]}{\partial t} & =-\left(k_{-1}+k_{2}\right)[C]+k_{1}[S][E], \\
\frac{\partial[P]}{\partial t} & =k_{2}[C] ;
\end{aligned}
$$

where $k_{1}, k_{-1}$ and $k_{2}$ are the reaction rates for the three reactions shown in eq.(3). Note that the total number of enzymes $[C]+[E]=\left[E_{0}\right]$ is conserved and that the product is removed immediately from the system and, therefore, the reverse reaction $C \leftarrow P+E$ is not considered. 
Assuming a quasi-steady approximation $\partial[C] / \partial t=0$, the steady concentration of the complex $C$ is derived from the equation of its evolution:

$$
[C]=\frac{[S]\left[E_{0}\right]}{K+[S]} ;
$$

where we have defined

$$
K=\frac{k_{-1}+k_{2}}{k_{1}} ;
$$

which can be used in eq.(4) to obtain the velocity of reaction as function of the concentration of the substrate:

$$
\frac{\partial[P]}{\partial t}=k_{2}\left[E_{0}\right] \frac{[S]}{K+[S]} ;
$$

within the condition $[S] \ll K$ we recover the prediction of the linear model, compare with eq.(2) using $k=k_{2}\left[E_{0}\right] / K$. For large values of the substrate concentration, $[S] \gg K$, the velocity of the reaction saturates to a maximum velocity $V_{\max }=k_{2}\left[E_{0}\right]$. For a comparison between the linear and the Michaelis-Menten models see Fig.1(c). While both types of dynamics coincide for small concentrations of the substrate, they differ for intermediate and large values.

\subsection{Goldbeter-Koshland model}

There are multiple examples of enzymatic reactions in cell biology, but two of the most characteristics are the phosphorylation and dephosphorylation of proteins. They are close related because the product of the first is the substrate for the second reaction and the product of the second is the substrate for the first reaction. The protein develops a reciprocal covalent modification [7].

The Goldbeter-Koshland model incorporates the enzymatic dynamics to the mechanism of phosphorylation and dephosphorylation. Therefore, assuming MichaelisMenten dynamics for both enzymes we arrive to the next set of biochemical reactions:

$$
M+K \text { in } \rightleftharpoons C_{1} \rightarrow M_{p}+K i n ;
$$

for the phosphorylation by an enzyme Kinase Kin of a protein $M$ into a phosphorylated protein $M_{p}$, and

$$
M_{p}+\text { Phos } \rightleftharpoons C_{2} \rightarrow M+\text { Phos; }
$$

for the dephosphorylation by an enzyme phosphatese Phos of a protein $M_{p}$. An sketch of both reactions is shown in Fig.(2). 


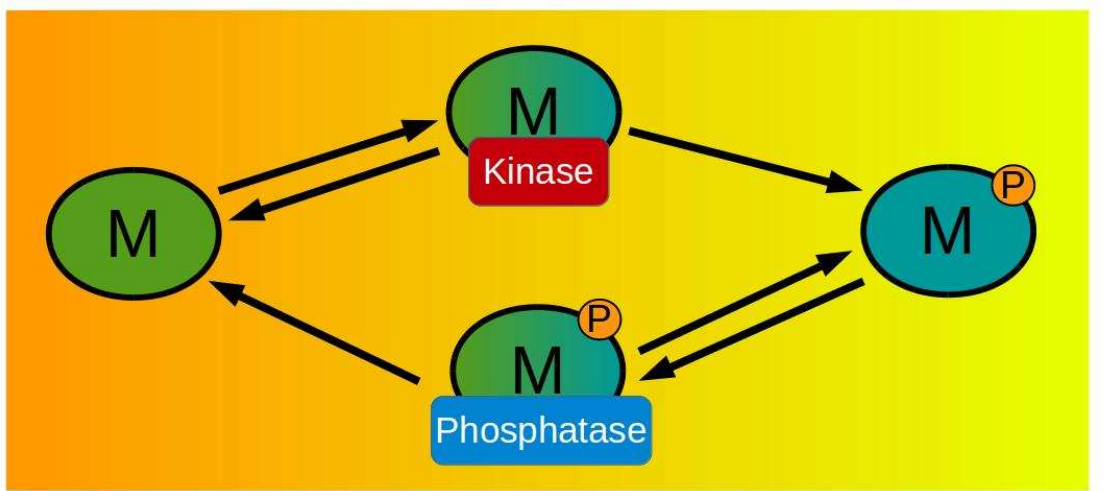

Fig. 2 Sketch of the reciprocal phosphorylation-dephosphorylation process of proteins. An enzyme kinase binds to the unphosphorylated protein to add a phosphate group. An enzyme phosphatase binds to the phosphorylated protein to remove the phosphate group.

Finally, the evolution of the concentrations of the two types of proteins $M$ and $M_{p}$ can be expressed with two non-linear equations after the assumption of quasi-static conditions for both complex $\partial\left[C_{1}\right] / \partial_{t}=0$ and $\partial\left[C_{2}\right] / \partial_{t}=0$ :

$$
\begin{gathered}
\frac{\partial[M]}{\partial t}=-G_{1} \frac{[M]}{K_{1}+[M]}+G_{2} \frac{\left[M_{p}\right]}{K_{2}+\left[M_{p}\right]} \\
\frac{\partial\left[M_{p}\right]}{\partial t}=+G_{1} \frac{[M]}{K_{1}+[M]}-G_{2} \frac{\left[M_{p}\right]}{K_{2}+\left[M_{p}\right]}
\end{gathered}
$$

with $G_{1}=k_{2}[\mathrm{Kin}]$ and $G_{2}=k_{4}[$ Phos $]$ for the kinase and phosphatase controlled reaction rates, and $K_{1}=\left(k_{-1}+k_{2}\right) / k_{1}$ and $K_{2}=\left(k_{-3}+k_{4}\right) / k_{3}$ for the equilibrium reactions. Furthermore, the total number of proteins is conserved: $[T]=[M]+\left[M_{p}\right]$.

The steady state condition is obtained when $\partial[M] / \partial t=0$ or equivalently $\partial\left[M_{p}\right] / \partial t=$ 0 . For a given set of parameter values only a single combination of values $[M]$ and $\left[M_{p}\right]$ is possible. It means that there is only a single solution.

With the tuning of a control parameter we may obtain large changes in the response. It permits the definition of two activation states. In this case, we consider $G_{1}$ as control parameter (equivalent analysis is possible with $G_{2}$ ), see for example Fig.3. Two different states are obtained corresponding to high concentration of $[M]$ or to high concentration of $\left[M_{p}\right]$. Depending on the relative activity between the kinase and the phosphatase, see Fig.3(a) and Fig.3(b), the solution of the steady state can be very different, see Fig.3(c-d). More important, the transition between these two states is not gradual but abrupt, a small change on the control parameter $G_{1}$ implies a big change in the response. This particular dynamics is employed to explain the activation of certain proteins in cell biology. For example, in the case of Fig.3, if we assume that the active form of the protein is $M_{p}$, for a value of $G_{1}=9$ (low values of $[k i n]$ ) the concentration of $M_{p}$ is small, however, if there is a slightly 
(a)

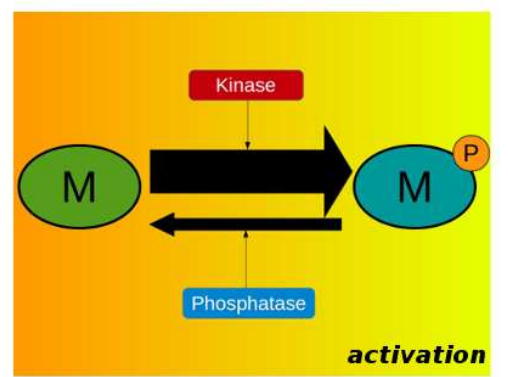

(b)

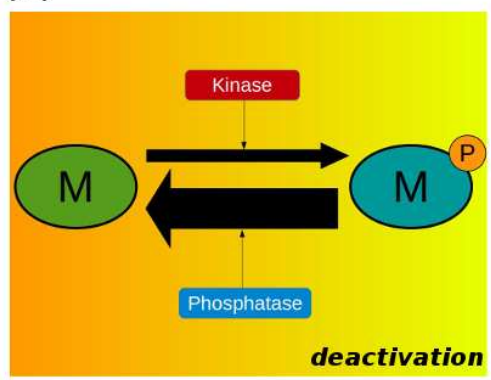

(c)

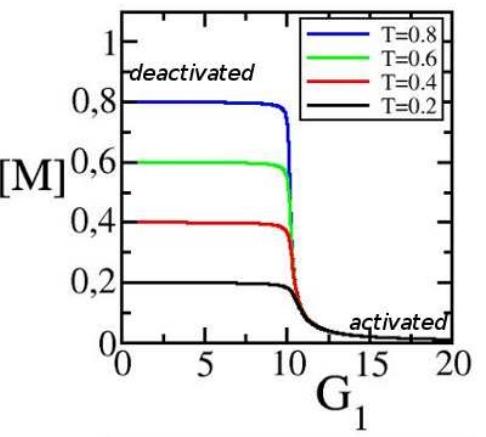

(d)

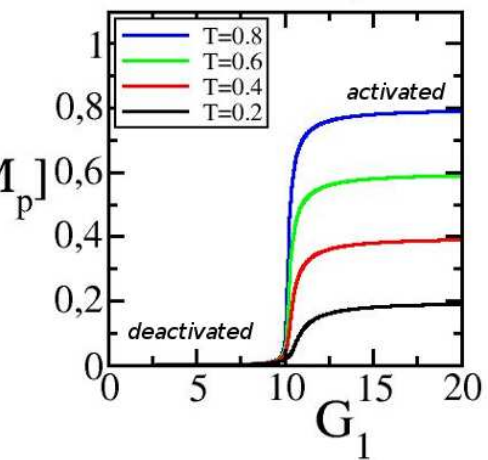

Fig. 3 (a) Sketch of a high rate of phosphorylation of a protein (large value of $G_{1}$ ). (b) Sketch of a low rate of phosphorylation of a protein (low value of $G_{1}$ ). (c-d) Dependence of the equilibrium concentration of the inactive (c) and the active (d) version of the protein in the value of the rate $G_{1}$ for different values of the total concentration of protein $\left(T=[M]+\left[M_{p}\right]\right)$.

increase of $[\mathrm{kin}]$ the parameter $G_{1}$ changes $\left(G_{1}=11\right)$ and the concentrations of $M_{p}$ strongly increases, which is the activate state in this example.

\subsection{Bistability and complex dynamics in protein activation}

The Goldbeter-Koshland mechanism produces a monotonous dependence on the response. Although the abrupt change in this dependence can be use as activation mechanism in cell signalling, more complex dynamics are needed for the explanation of some other observations in cell biology [2].

Interactions among enzymes, substrates and products may induce a bistable switch [13]. For example, a positive feedback of the phosphorylated protein $M_{p}$ in the enzyme kinase, modifies the Michaelis-Menten kinetics into a system with higher order terms:

$$
\frac{\partial[M]}{\partial t}=-G_{1} \frac{[M]}{K_{1}+[M]}\left(1+A \frac{\left[M_{p}\right]}{K_{B}+\left[M_{p}\right]}\right)+G_{2} \frac{\left[M_{p}\right]}{K_{2}+\left[M_{p}\right]},
$$


a)

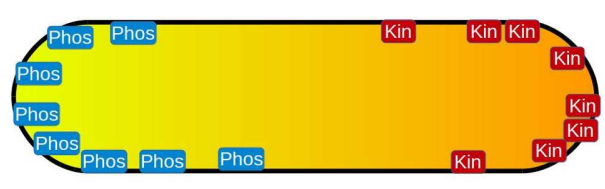

b)

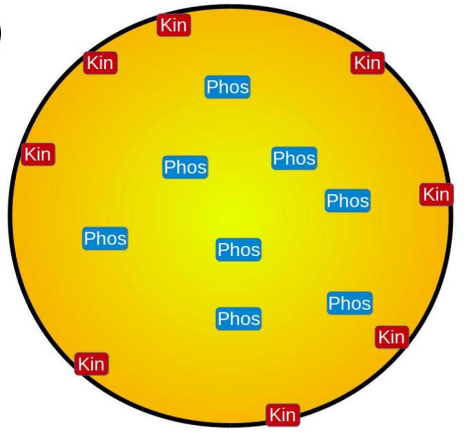

Fig. 4 Inhomogeneous affinity of enzymes. (a) Location of kinases and phosphatases at opposite poles in bacteria. (b) Location of kinases at cellular membrane and of phosphatases in the cytosol in Eukaryote.

$$
\frac{\partial\left[M_{p}\right]}{\partial t}=+G_{1} \frac{[M]}{K_{1}+[M]}\left(1+A \frac{\left[M_{p}\right]}{K_{B}+\left[M_{p}\right]}\right)-G_{2} \frac{\left[M_{p}\right]}{K_{2}+\left[M_{p}\right]} ;
$$

which induces bistability for a wide parameter range.

Although this interaction are observed in some experimental cases, equivalent terms are also obtained due to a saturation at the different compartments of the cell, as we discuss in the following sections.

\section{Spatial aspects of enzymatic kinetics}

The interior of living cells is outside of the well-mixed approach because the cytoplasm is heterogeneous. Therefore, the concentrations of enzymes and proteins are not be homogeneous. Proteins may have tendency to accumulate in some parts of the cell. A typical example of this heterogeneous distributions is the effect of membranes. Enzymes can interact with the membranes and accumulate, for example in opposite regions of a bacteria [16], see Fig.4(a). A different case corresponds to the accumulation at the membrane of only one type of enzyme, for example the kinases in Fig.4(b), such inhomogeneous distribution induces a gradient between the interior and the exterior of the cytoplasm [13].

On the other hand, the spatial location of the protein implies important limitations in the protein distribution. For example, the space at the membrane is limited because of the high density of proteins and structures.

To model the effects of the compartments in the Goldbeter-Koshland mechanism, first, we introduce a constrain related with the accessible space at the membrane in the reaction equations for the protein concentrations. Second, we consider the intermediate concentration to account for the time of the unphosphorylated protein to diffuse to the membrane. 


\subsection{Saturation at the membrane}

The cellular membrane is a busy environment where the addition of large quantity of a new protein may occupy an extended region of the available area at the inner part of the membrane. It incorporates an extra constrain in the modeling of the dynamics of the protein concentration because the saturation of the membrane prevents the binding of new proteins from the cytoplasm.

\subsubsection{Membrane-controlled binding}

If the concentration of protein at the membrane approaches the saturation concentration, the binding rate decreases to zero. Assuming that the concentration of kinases is low, we renormalized the binding rate $G_{2}$ with a factor which accounts for the available space.

$$
\begin{aligned}
\frac{\partial[M]}{\partial t} & =-G_{1} \frac{[M]}{K_{1}+[M]}+G_{2}\left(1-\frac{[M]}{\left[M_{S}\right]}\right) \frac{\left[M_{p}\right]}{K_{2}+\left[M_{p}\right]}, \\
\frac{\partial\left[M_{p}\right]}{\partial t} & =+G_{1} \frac{[M]}{K_{1}+[M]}-G_{2}\left(1-\frac{[M]}{\left[M_{S}\right]}\right) \frac{\left[M_{p}\right]}{K_{2}+\left[M_{p}\right]} ;
\end{aligned}
$$

where $M_{S}$ is the saturation concentration at the membrane. If $[M]=\left[M_{S}\right]$ the membrane is full, the prefactor $\left(1-[M] /\left[M_{S}\right]\right)$ is zero, and new proteins cannot bind to the membrane.

The linear stability analysis of eqs.(12) shows the existence of three different solutions. However, for a give value of the control parameter $G_{1}$ only one of the three solutions is stable. Therefore, there is a monotonous behaviour on the control parameter, see Fig.5(a). In the limit $G_{1} \rightarrow 0$ the concentration of proteins at the membrane approaches to $[M]=T$ for $T<\left[M_{S}\right]$, e.g. all the proteins are at the membrane, or to $[M]=\left[M_{S}\right]$ for $T>\left[M_{S}\right]$, because not all proteins can bind to the membrane. In the opposite limit, large values of $G_{1}$, the membrane is empty $[M]=0$.

\subsubsection{Membrane-controlled reaction}

A saturated membrane precludes the binding of all types of proteins including the enzymes. The high concentration of membrane-bound proteins incorporates an additional constrain to the binding of the kinase at the membrane. In such case, the unbinding rate of $M$, related with the enzymatic reaction leaded by the kinase, incorporates an equivalent constrain term than the binding rate, see the following set of nonlinear equations for the concentrations:

$$
\frac{\partial[M]}{\partial t}=-G_{1}\left(1-\frac{[M]}{\left[M_{S}\right]}\right) \frac{[M]}{K_{1}+[M]}+G_{2}\left(1-\frac{[M]}{\left[M_{S}\right]}\right) \frac{\left[M_{p}\right]}{K_{2}+\left[M_{p}\right]},
$$



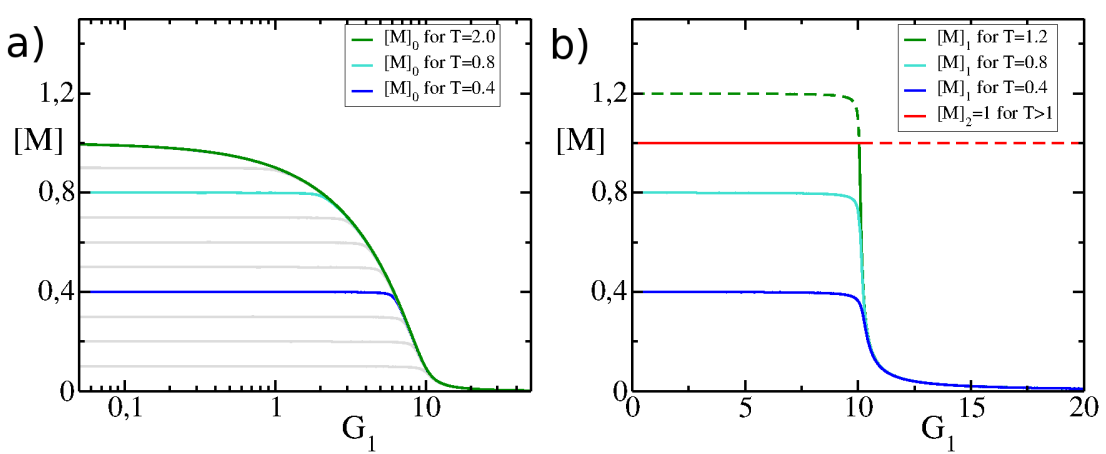

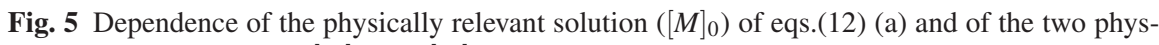
ically relevant solutions $\left([M]_{1}\right.$ and $[M]_{2}$ ) of eqs.(13) (b) of the inactive version of the protein in the value of the rate $G_{1}$ for different values of the total concentration of protein $\left(T=[M]+\left[M_{p}\right]\right)$. Dashed lines correspond to unphysical or unstable solutions.

$$
\frac{\partial\left[M_{p}\right]}{\partial t}=+G_{1}\left(1-\frac{[M]}{\left[M_{S}\right]}\right) \frac{[M]}{K_{1}+[M]}-G_{2}\left(1-\frac{[M]}{\left[M_{S}\right]}\right) \frac{\left[M_{p}\right]}{K_{2}+\left[M_{p}\right]} ;
$$

where $M_{S}$ is the saturation concentration at the membrane. The high concentration of proteins at the membrane inhibits both binding and unbinding by phosphorylation of proteins.

The linear stability analysis of eqs.(13) reveals the existence of two physical solutions. One of the solutions corresponds to the complete saturation of the membrane with $[M]_{2}=M_{S}$. Increasing the control parameter $G_{1}$, the complete saturation condition becomes unstable and the new stable solution with $[M]_{1}<M_{S}$ decreases to $[M]_{1}=0$ at large values of $G_{1}$. The combination of the two stable solutions produces a monotonous response and no bistability is obtained.

In Fig.5(b) three cases are studied for different values of $T$, keeping $M_{S}=1$. If $T<1$ there is a unique solutions $[M]_{1}$ for all the values of $G_{1}$. In this case, for $G_{1}<10$ the value of $[M]_{1}$ saturates to $M=T$. Such saturation is unphysical for $T>1$ and the solution $M=T$ is not possible. In this case, the second solution $[M]_{2}=M_{S}=1$ is stable for $G_{1}<10$ and exchanges stability with the the solution $[M]_{1}$ at $G_{1}=10$. For large values of $G_{1}$ the solutions $[M]_{2}=M_{S}$ is not stable, see Fig.5(b).

\subsection{Cytosolic diffusion}

The models previously discussed relate unphosphorylated proteins to the membrane and phosphorylated proteins to the cytoplasm. With these assumptions we neglect the concentration of phosphorylated proteins at the membrane and unphosphorylated proteins in the cytosol. The first assumption seems adequate because there is an immediate lose of affinity of the proteins to the membrane after phosphoryla- 


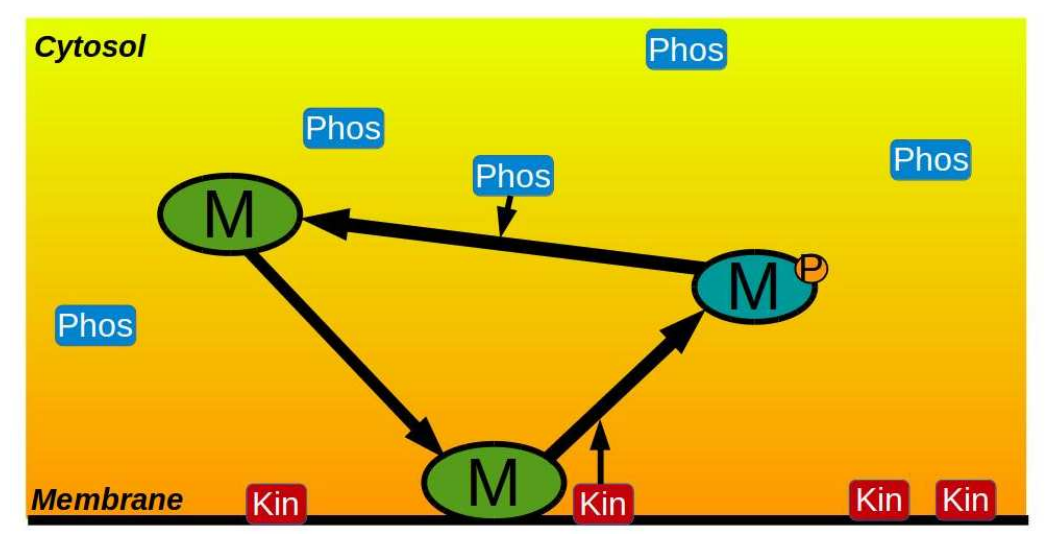

Fig. 6 Cyclic dynamics of the protein, from the membrane to the cytoplasm, phosphorylation, and from the cytoplasm back to the membrane.

tion. However, after the dephosphorylation reaction the resulting protein needs to diffuse to the membrane and the binding to the membrane is not immediate. A third concentration of unphosphorylated cytosolic protein $\left[M_{c}\right]$ can be considered.

In summary, the protein is translocated from the membrane when it is phosphorylated by a kinase. Back in the cytoplasm, the translocated proteins are dephosphorylated by a phosphatase. The resulting unphosphorylated proteins diffuse and bind again at the membrane. These three processes give rise to a cyclic dynamics, see Fig.6. We derive the next set of equations for the three concentrations:

$$
\begin{aligned}
& \frac{\partial[M]}{\partial t}=-G_{1}\left(1-\frac{[M]}{\left[M_{S}\right]}\right) \frac{[M]}{K_{1}+[M]}+G_{3}\left(1-\frac{[M]}{\left[M_{S}\right]}\right)\left[M_{c}\right], \\
& \frac{\partial\left[M_{p}\right]}{\partial t}=+G_{1}\left(1-\frac{[M]}{\left[M_{S}\right]}\right) \frac{[M]}{K_{1}+[M]}-G_{2} \frac{\left[M_{p}\right]}{K_{2}+\left[M_{p}\right]}, \\
& \frac{\partial\left[M_{c}\right]}{\partial t}=-G_{3}\left(1-\frac{[M]}{\left[M_{S}\right]}\right)\left[M_{c}\right]+G_{2} \frac{\left[M_{p}\right]}{K_{2}+\left[M_{p}\right]}
\end{aligned}
$$

where $G_{3}$ is the binding rate to the membrane of the cytosolic proteins. We assume that the affinity to the membrane is linear on $\left[M_{c}\right]$ and it is penalized by a possible saturation of the membrane. Note also that the total number of proteins is conserved $[M]+\left[M_{c}\right]+\left[M_{p}\right]=T$, giving rise to a mass-conserved model [25].

The linear stability analysis of eqs.(14) shows the simultaneous existence of three different physically relevant steady solutions for a window of values of the control parameter. There is non-monotonous dependence on the parameter $G_{1}$ and bistability appears, see Fig.7.

For a total number of proteins below the saturation value $T<\left[M_{S}\right]$ there is only one solution and its dependence on $G_{1}$ is monotonous, see Fig.7(a). The concentration at the membrane decreases to zero for large values of $G_{1}$. 
a)

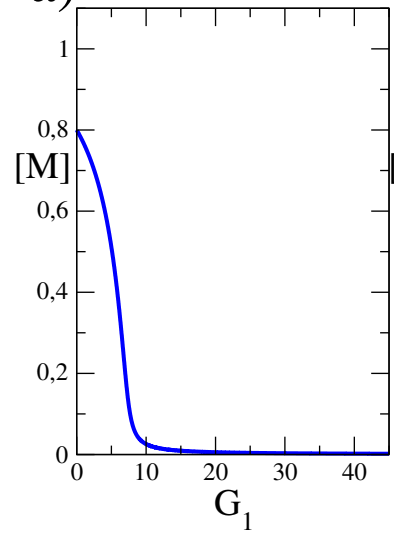

b)

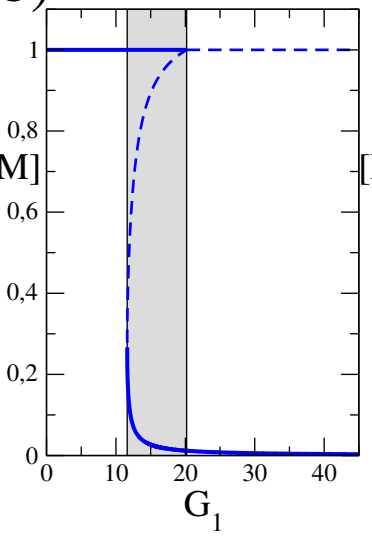

c)

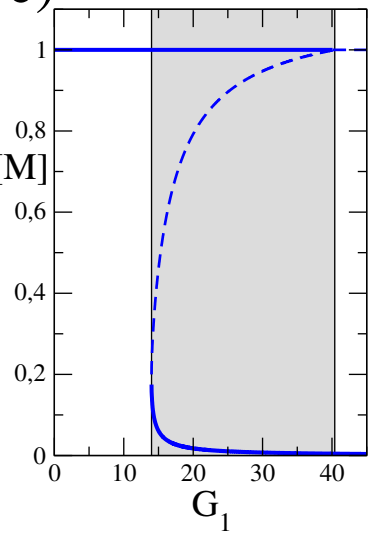

Fig. 7 Dependence of the physically relevant solutions of the inactive version of the protein in the value of the rate $G_{1}$ for different values of the total concentration of protein $\left(T=[M]+\left[M_{p}\right]+\right.$ $\left.\left[M_{c}\right]\right): T=0.8(\mathrm{a}), T=1.2(\mathrm{~b})$, and $T=1.4$ (c). Solid and dashed lines correspond, respectively, to stable and unstable solutions. Gray areas mark region of bistability where two possible values of $[\mathrm{M}]$ are stable.

For $T>\left[M_{S}\right]$ a new solution is possible. It consists in a completely saturated membrane, full of proteins $[M]=\left[M_{s}\right]$, and the excess of proteins are located in the cytoplasm, see Fig.7(b,c). It produces a bistable condition for a window of values of the parameter $G_{1}$. The saturation condition for the membrane is not stable for large values of $G_{1}$, see Fig.7(b,c).

\section{Reaction-diffusion model of phosphorylation and dephosphorylation of proteins}

Now, we explicitly consider the spatial distribution of the proteins with the use of spatio-temporal equations for the three concentrations. We take into account the diffusion $\left(D_{c}\right)$ of the unphosphorylated $\left(M_{c}\right)$ and phosphorylated $\left(M_{p}\right)$ proteins at the cytoplasm and the diffusion of the unphosphorylated bound proteins at the membrane $\left(D_{m}\right)$. The set of reaction-diffusion equations read:

$$
\begin{aligned}
& \frac{\partial[M]}{\partial t}=-G_{1}\left(1-\frac{[M]}{\left[M_{S}\right]}\right) \frac{[M]}{K_{1}+[M]}+G_{3}\left(1-\frac{[M]}{\left[M_{S}\right]}\right)\left[M_{c}\right]+\nabla \cdot D_{m} \nabla[M], \\
& \frac{\partial\left[M_{p}\right]}{\partial t}=+G_{1}\left(1-\frac{[M]}{\left[M_{S}\right]}\right) \frac{[M]}{K_{1}+[M]}-G_{2} \frac{\left[M_{p}\right]}{K_{2}+\left[M_{p}\right]}+\nabla \cdot D_{c} \nabla[M] \\
& \frac{\partial\left[M_{c}\right]}{\partial t}=-G_{3}\left(1-\frac{[M]}{\left[M_{S}\right]}\right)\left[M_{c}\right]+G_{2} \frac{\left[M_{p}\right]}{K_{2}+\left[M_{p}\right]}+\nabla \cdot D_{c} \nabla[M]
\end{aligned}
$$


a)

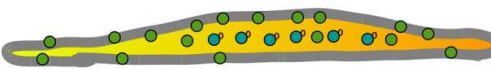

b)

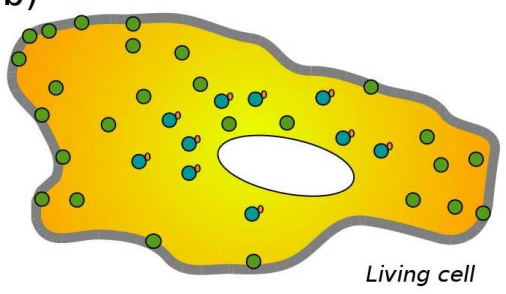

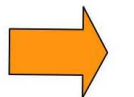
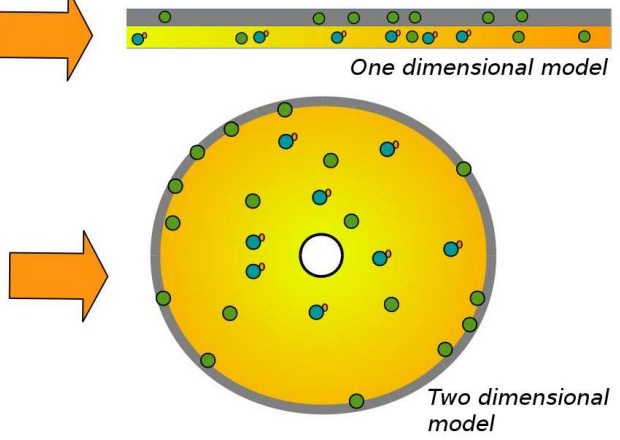

Fig. 8 Geometry reduction for the implementation of the model on Pattern formation inside living cells. (a) Reduction to a simple one dimensional geometry using to different values for the concentration at the membrane and in the cytoplasm. (b) Simplification of the cell to symmetric two-dimensional geometry with no-flux boundary conditions and binding to the membrane.

where the diffusion of the proteins in the cytosol is higher than at the membrane $\left(D_{m}<<D_{c}\right)$. The characteristic values of the diffusion coefficient in the cytosol are around two orders of magnitude larger than the value of a equivalent molecule at the membrane [31].

For the integration of eqs.(15) we have to define adequate boundary conditions. One possibility is the use of non-flux boundaries in a one-dimensional approach of the cell, see Fig.8(a). This type of models are commonly used for the description of cell polarity [20, 23, 19, 25]. We employ this model for the calculation of the linear stability analysis in Sec.4.1. On the other hand, we can employ the cell membrane as boundary conditions in a two-dimensional domain, see Fig.8(b). Such type of models has been employed to simulate insulin-secreting cells [27] and in polarity of yeast cell [21]. We use such approach to perform the numerical simulations appearing in Sec.4.2 and 4.3.

\subsection{Linear stability analysis}

The number of homogeneous solutions depends on the parameter values. Changing the parameters $T$ and $G_{1}$ two different zones appear: a region where a single solution is possible and a region where three solutions appear. To study its stability we calculate the linear stability analysis of eqs.(15). For a particular homogeneous steady state, composed by the concentrations $[M]_{0},\left[M_{c}\right]_{0}$, and $\left[M_{p}\right]_{0}$, we introduce a perturbation:

$$
\begin{aligned}
{[M] } & =[M]_{0}+(\delta M) e^{\omega t+i k x}, \\
{\left[M_{c}\right] } & =\left[M_{c}\right]_{0}+\left(\delta M_{c}\right) e^{\omega t+i k x},
\end{aligned}
$$




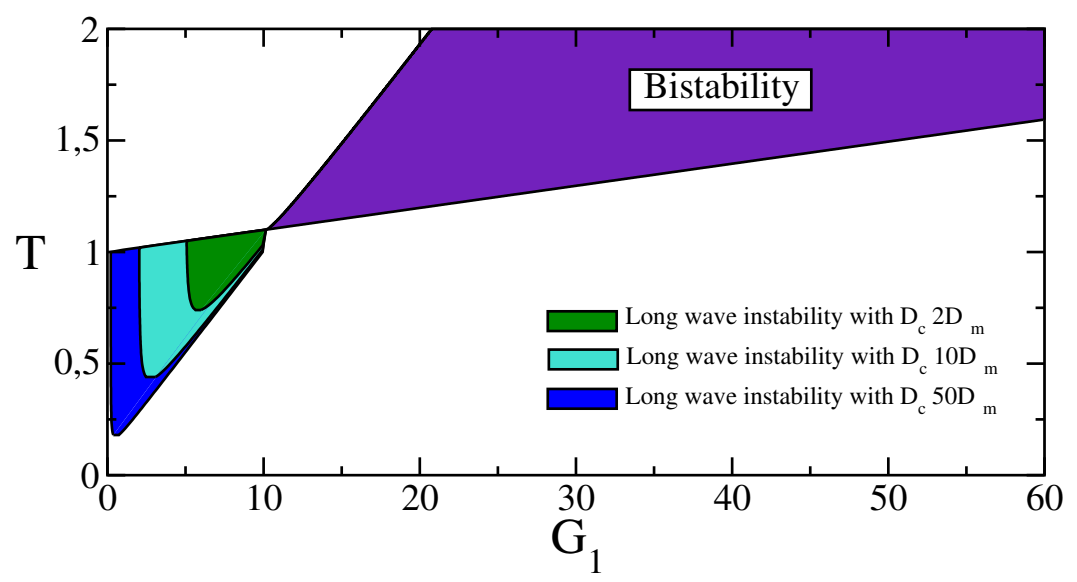

Fig. 9 Analytic phase diagram of the reaction-diffusion system, see eqs.(15). The linear stability problem of the homogeneous solutions is solved in the parameter space defined by $G_{1}$ and $T$ for three different ratios $D_{m} / D_{c}$.

$$
\left[M_{p}\right]=\left[M_{p}\right]_{0}+\left(\delta M_{p}\right) e^{\omega t+i k x},
$$

and evaluate if the perturbations grow or decrease with time using eqs.(15). The variable $\omega$ is the growing rate and indicates the stability of the solution to small perturbations.

The results of the linear stability analysis of eqs.(15) is plotted in Fig.9. It shows a region where only two of the three solutions are stable (bistability), corresponding to the situation described in the previous section 3.2, and a region where the unique physically relevant solution is unstable due to a long-wave instability. This instability produces spontaneous domain formation and, because of the conserved protein concentration, the posterior coarsening into a reduced number of domains.

While bistability is independent of the diffusion coefficients, the long wave instability appears initially at a given value of wavenumber which will depend on the quotient $D_{m} / D_{c}$. The area of the phase diagram where the solution is unstable changes with the diffusion as it is shown in Fig.9 for three values of the quotient $D_{m} / D_{c}$.

Two different types of pattern formation, with different dynamics, are expected depending on the parameter values. Next we analyze separately both mechanisms.

\subsection{Long-wave instability}

For the parameter values inside the region of long-wave instability, a one-dimensional system, see Sketch in Fig.8(a), spontaneously develops the formation of domains as predicted by the linear stability analysis. If we change the symmetry of the integration domain, see Sketch in Fig.8(b), the linear stability analysis shown in the previ- 
a)

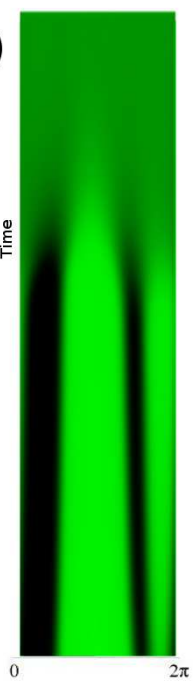

b) Profile

$$
\Sigma^{\equiv}
$$

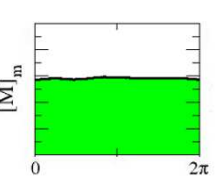

C)

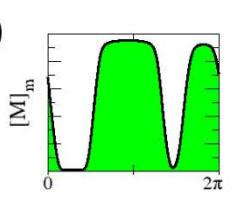

d)

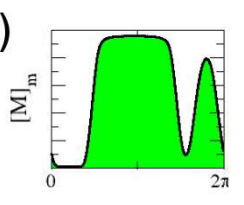

$[\mathrm{M}] \mathrm{m}$
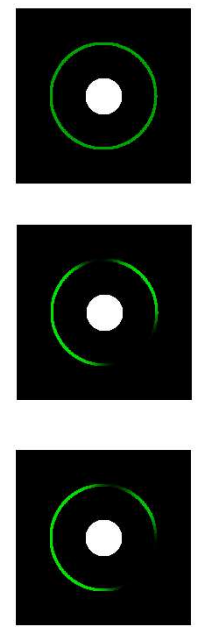

$[\mathrm{M}] \mathrm{c}$
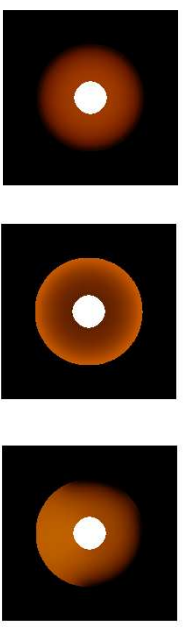

$[\mathrm{M}] \mathrm{p}$
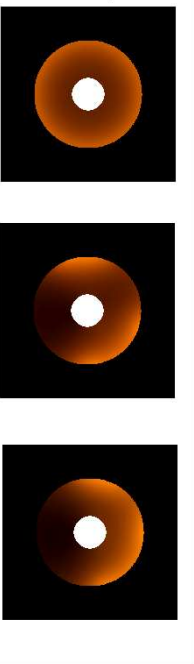

Fig. 10 Pattern formation by the a long wave instability mechanism. A circular domain is employed, representing the interior and the membrane of a living cell. (a) Spatio-temporal plot of the concentration of membrane-bound protein. (b-d) Profiles of the membrane protein and spatial distribution of the membrane-bound, cytosolic unphosphorylated and phosphorylated protein at times: $t=0(\mathrm{~b}), t=t_{\text {tot }} / 2$ (c), and $t=t_{\text {tot }}(\mathrm{d})$, where $t_{\text {tot }}$ is the total time of the numerical simulation.

ous section cannot be applied directly. However, it is known [26] that the parameter values can be renormalized considering the size of the two-dimensional cytoplasm in comparison with the one-dimensional membrane.

Numerical simulations for a convenient choice of the parameter values are shown in Fig.10. First, the spatio-temporal plot in Fig.10(a) shows the evolution of the concentration of membrane-bound proteins. From an initially homogeneous condition with a small spatially distributed random perturbation, two evolving maxima appear. While one of the domains grows the other one decreases, and finally, only one single large domain survives. Such competition among the domains is a typical signature of coarsening.

In panel (b) of Fig.10 the initial condition is plotted, an homogeneous concentration with a small random perturbation around the unstable value. Two-dimensional panels with the distribution of free protein concentration and phosphorylated concentration at the membrane and in the cytosol are also shown.

In the other two panels (c) and (d) of Fig.10 the spatial distributions of the concentrations are shown at two different times. Note that the concentrations of $[M]_{c}$ and $[M]_{p}$ are complementary: large (small) values of $[M]_{c}$ coincide with small (large) $[M]_{p}$. Furthermore, $[M]_{p}$ accumulates in the region of the cytoplasm close to regions of the membrane where no proteins are bound. 
a)

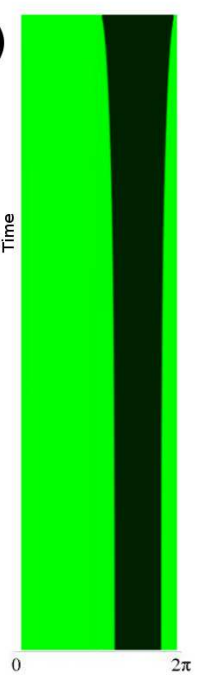

b)

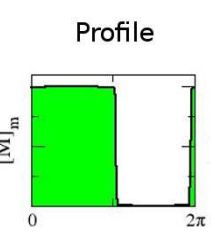

$[\mathrm{M}] \mathrm{m}$

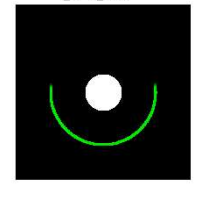

C)

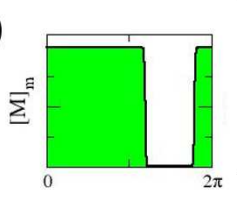

d)
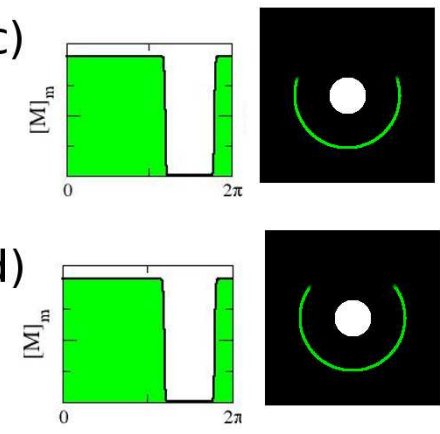
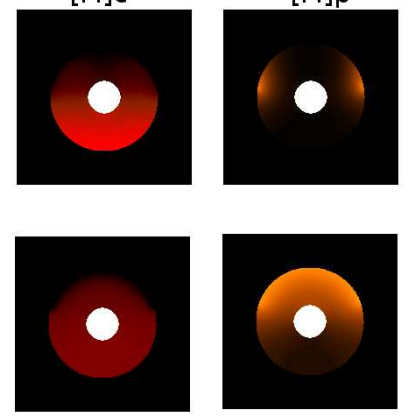
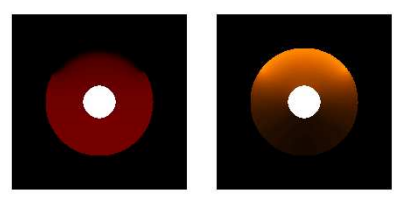

Fig. 11 Pattern formation by the bistable-induced mechanism. A circular domain is employed, representing the interior and the membrane of a living cell. (a) Spatio-temporal plot of the concentration of membrane-bound protein. (b-d) Profiles of the membrane protein and spatial distribution of the membrane-bound, cytosolic unphosphorylated and phosphorylated protein at times: $t=0$ (b), $t=t_{\text {tot }} / 2$ (c), and $t=t_{\text {tot }}(\mathrm{d})$, where $t_{\text {tot }}$ is the total time of the numerical simulation.

\subsection{Bistability-induced instability}

Numerical simulations in the bistable region of the parameter space produce similar final domains than numerical results shown in the previous section, see a representative example in Fig.11. However, the mechanism and the conditions are different. Under bistability the two homogeneous solution are stable, and, therefore, an small perturbation of the homogeneous solution decreases, and eventually, the homogeneous condition is recovered.

However, if the two solutions are connected by a front, see Fig.11(b), it moves as it is shown in the spatio-temporal plot in Fig.11(a). In contrast to a classical bistable system, here there is a mass-conserved condition which precludes the complete translocation to the membrane of the proteins in the cytoplasm. Finally, the two solutions are separated by an pinned wave [23], see Fig.11(c) and (d).

\section{Conclusions}

We employ an extended version of the classical Goldbeter-Koshland model on covalent modification in biological systems for phosphorylation and dephosphorylation of proteins. From a simple activation model based in two opposite enzymatic reac- 
tions following Michaelis-Menten kinetics, we have generated a scenario for pattern formation in the interior of a living cell. The mechanism is based on the different localization inside the cell of the two enzymatic reactions: while phosphorylation only occurs at the membrane of the cell, the opposite reaction occurs in the cytoplasm. After dephosphorylation the resulting proteins diffuse to the membrane where they bind and the cycle can start again.

We analyze two different mechanisms of pattern formation for protein at membranes: A long wave instability and a bistability-related mechanism, previously described in models of cell polarization [20] and [23] respectively. The conditions of the model to fulfill the requirements for pattern formation are simple and generic:

- Difference on the diffusion coefficients. The first important condition is the large diffusion in the cytoplasm in comparison with the diffusion in the membrane $D_{c} \gg D_{m}$ [31]. This constrain is naturally achieved by living cells where diffusion of proteins in the cytoplasm is estimated to be around $D_{c} \sim 1-10 \mu \mathrm{m}^{2} / \mathrm{s}$ [32], while membrane diffusion coefficient of proteins in mammalian cells has been estimated to be $D_{m} \sim 0.1 \mu \mathrm{m}^{2} / \mathrm{s}$ [33].

- Compartmentalization of the reaction. The separation of the two enzymatic processes in two different compartments of the cell (phosphorylation at the membrane and dephosphorylation in the cytoplasm) introduces a delay between dephosphorylation and the re-binding of the protein to the membrane. This temporal delay accounts for the diffusion time of the proteins in the cytoplasm.

- Mass-conservation. The conservation of the total number of proteins is an important condition for the bistability-related mechanism, this constrain stops the bistable front going from the metastable to a stable solution [23].

Similar extensions of the Goldbeter-Koshland mechanism may be applicable to a large variety of biological systems. Phosphorylation by kinases regulates multiple processes in living cells, e.g. the formation of polarity of cells induced by PAR proteins [19], the cyclic dynamics of Rho GTPases [20] or the regulation of the cell division of E. Coli controlled by the Min proteins [36].

The approach described here has been employed in the modeling of the myristoylelectrostatic switch [9] composed by a cyclic binding and unbinding dynamics of the myristoylated alanine-rich C kinase substrate proteins (MARCKS). After phosphorylation of MARCKS proteins by protein kinase C (PKC), MARCKS proteins lose their affinity to membranes. The phosphates reduce the positive charge of the protein and cause the unbinding from the membrane. In the cytoplasm, phosphatases remove the phosphates from the protein and, consequently, MARCKS can bind again at the membrane. This system has been recently described in terms of massconserved reaction-diffusion equations [26, 27] and the resulting equations have been employed for the calibration of the binding of the MARCKS at lipid monolayers [28, 29].

In our approach stochastic effects due to a low number of proteins have been neglected. The typical large concentration of proteins in cells permits the use of deterministic dynamics, however, the concentration of enzymes is smaller and stochastic 
effects may become relevant. The use of a stochastic model may enhance domain formation $[34,35]$.

Living cells are three-dimensional and future models will take this condition into account. The mechanisms are, however, equivalent at different levels of spatial complexity. One-dimensional approximation is employed to calculate linear stability analysis and identify the instabilities. The two-dimensional view is, however, sufficient to perform numerical simulations and describe proteins concentrations at the membrane and in the different regions of the cytoplasm, see Fig.10 and Fig.11. Such two-dimensional modeling considers the cytoplasm volume and diffusion orthogonal to the membrane. The proteins can diffuse from the membrane and are, hence, diluted near the membrane. As the cytosolic volume increases, the concentration of proteins close to the membrane decreases and the binding process is affected. However, the change of the cytosolic volume is equivalent to a renormalization of some of the reaction rates in the model [26].

In summary, we have developed a simple model of binding, phosphorylation and desphosphorylation for membrane proteins, which predicts the spontaneous appearance of domains of high protein concentration at the membrane of living cells.

Acknowledgements I acknowledge fruitful discussions with professor Markus Bär. Support by MINECO of Spain under the Ramon y Cajal program with the grant number RYC-2012-11265 is acknowledged.

\section{References}

1. Alberts B, Johnson A, Lewis J, Raff M, Roberts K, Walter P: Molecular Biology of the Cell. Garland Science, New York (2002)

2. Tyson, J. J., Chen, K. C., Novak, B.: Sniffers, buzzers, toggles and blinkers: dynamics of regulatory and signaling pathways in the cell. Current opinion in cell biology, 15, 221-231 (2003)

3. Novák, B., Tyson, J. J.: Design principles of biochemical oscillators. Nature reviews Molecular cell biology, 9, 981-991 (2008)

4. Tyson, J. J., Novák, B.: Functional motifs in biochemical reaction networks. Annual review of physical chemistry, $\mathbf{6 1}, 219$ (2010)

5. Keener, J., Sneyd, J.: Mathematical Physiology. Springer, New York (2009).

6. Kholodenko, B. N., Hancock, J. F. and Kolch, W.: Signalling ballet in space and time. Nature reviews Molecular cell biology, 11, 414-426 (2010)

7. Goldbeter, A., Koshland, D. E.. An amplified sensitivity arising from covalent modification in biological systems. Proceedings of the National Academy of Sciences 78, 6840-6844 (1981)

8. Straube, R., Conradi, C.: Reciprocal enzyme regulation as a source of bistability in covalent modification cycles. Journal of theoretical biology, 330, 56-74 (2013)

9. Bressloff, P. C., Newby, J. M.: Stochastic models of intracellular transport. Reviews of Modern Physics, 85, 135 (2013)

10. Dehmelt, L., Bastiaens, P. I.: Spatial organization of intracellular communication: insights from imaging. Nature Reviews Molecular Cell Biology, 11, 440-452 (2010)

11. Karsenti, E.: Self-organization in cell biology: a brief history. Nature Reviews Molecular Cell Biology, 9, 255-262 (2008)

12. Kholodenko, B. N., Hoek, J. B., Westerhoff, H. V.: Why cytoplasmic signalling proteins should be recruited to cell membranes. Trends in cell biology, 10, 173-178 (2000) 
13. Kholodenko, B. N.: Cell-signalling dynamics in time and space. Nature reviews Molecular cell biology, 7, 165-176 (2006)

14. Maeder, C. I., Hink, M. A., Kinkhabwala, A., Mayr, R., Bastiaens, P. I., Knop, M.: Spatial regulation of Fus3 MAP kinase activity through a reaction-diffusion mechanism in yeast pheromone signalling. Nature Cell Biology, 9, 1319-1326 (2007)

15. Suzuki, A., Ohno, S.: The PAR-aPKC system: lessons in polarity. Journal of cell science, 119 , 979-987 (2006)

16. Tropini, C., Rabbani N., and Huang K. C.: Physical constraints on the establishment of intracellular spatial gradients in bacteria. BMC biophysics 5, 17 (2012)

17. Mogilner, A., Allard J., Wollman R.: Cell polarity: quantitative modeling as a tool in cell biology. Science 336, 175-179 (2012)

18. Van Haastert, P. J., Devreotes, P. N.: Chemotaxis: signalling the way forward. Nature reviews Molecular cell biology, 5, 626-634 (2004)

19. Goehring, N. W., P. K. Trong, J. S. Bois, D. Chowdhury, E. M. Nicola, A. A. Hyman, and S. W. Grill. Polarization of PAR proteins by advective triggering of a pattern-forming system. Science 334, 1137-1141 (2011)

20. Otsuji, M., S. Ishihara, K. Kaibuchi, A. Mochizuki, and S. Kuroda. A mass conserved reaction-diffusion system captures properties of cell polarity. PLoS computational biology 3, e108 (2007)

21. Goryachev, A. B., and A. V. Pokhilko. Dynamics of Cdc42 network embodies a Turing-type mechanism of yeast cell polarity. FEBS letters 582, 1437-1443 (2008)

22. Orlandini, E., D. Marenduzzo, and A. B. Goryachev. Domain formation on curved membranes: phase separation or Turing patterns?. Soft Matter 9, 9311-9318 (2013)

23. Mori, Y., A. Jilkine, and L. Edelstein-Keshet. Wave-pinning and cell polarity from a bistable reaction-diffusion system. Biophysical journal 94, 3684-3697 (2008)

24. Beta, C., Amselem, G., Bodenschatz, E.: A bistable mechanism for directional sensing. New Journal of Physics, 10, 083015 (2008)

25. Trong, P. K., E. M. Nicola, N. W. Goehring, K. V. Kumar, and S. W. Grill. Parameter-space topology of models for cell polarity. New Journal of Physics 16, 065009 (2014)

26. Alonso, S., and M. Bär. Phase separation and bistability in a three-dimensional model for protein domain formation at biomembranes. Physical biology 7, 046012 (2010)

27. Alonso, S., and M. Bär. Modeling domain formation of MARCKS and protein kinase $\mathrm{C}$ at cellular membranes. EPJ Nonlinear Biomedical Physics 2, 1-18 (2014)

28. Alonso, S., U. Dietrich, C. Händel, J. A. Käs, and M. Br. Oscillations in the lateral pressure of lipid monolayers induced by nonlinear chemical dynamics of the second messengers MARCKS and protein kinase C. Biophysical journal 100, 939-947 (2011)

29. Lippoldt, J., Händel, C., Dietrich, U., Käs, J. A.: Dynamic membrane structure induces temporal pattern formation. Biochimica et Biophysica Acta (BBA)-Biomembranes, 1838, 23802390 (2014)

30. Mogami, H., Zhang, H., Suzuki, Y., Urano, T., Saito, N., Kojima, I., Petersen, O. H.: Decoding of short-lived $\mathrm{Ca} 2+$ influx signals into long term substrate phosphorylation through activation of two distinct classes of protein kinase C. Journal of Biological Chemistry, 278, 9896-9904 (2003)

31. Edidin M, Zagyansky Y, Lardner TJ: Measurement of membrane protein lateral diffusion in single cells. Science, 191466468 (1976)

32. Luby-Phelps K: Cytoarchitecture and physical properties of cytoplasm: volume, viscosity, diffusion, intracellular surface area. Int Rev Cytol, 192, 189221 (1997)

33. Valdez-taubas J, Pelham RB: Slow diffusion of proteins in the yeast plasma membrane allows polarity to be maintained by endocytic cycling. Curr Biol, 1316361640 (2003).

34. Lawson, M. J., Drawert, B., Khammash, M., Petzold, L., Yi, T. M.: Spatial stochastic dynamics enable robust cell polarization. PLoS computational biology, 9, e1003139 (2013)

35. McKane, A. J., Biancalani, T., Rogers, T.: Stochastic pattern formation and spontaneous polarisation: the linear noise approximation and beyond. Bulletin of mathematical biology, 76, 895-921 (2014)

36. Howard M., Kruse K.: Cellular organization by self-organization:mechanisms and models for Min protein dynamics. J Cell Biol, 168, 53336 (2005) 